\title{
Longitudinal changes in objectively measured physical activity differ for weekdays and weekends among Chinese children in Hong Kong
}

\author{
Stephen Heung-Sang Wong ${ }^{1}$, Wendy Yajun Huang ${ }^{2^{*}}$ and Gang He ${ }^{1}$
}

\begin{abstract}
Background: Cross-sectional investigation showed that Chinese children in Hong Kong were more physically active on weekends than weekdays, which is contrary to previous findings. However, little is known as to whether these time-segment-specific differences persist with age. This study aimed to compare the 2-year changes in accelerometer-assessed physical activity (PA) and sedentary time (ST) between weekdays and weekends among Chinese children in Hong Kong.

Methods: Children aged 6-8 years were recruited from primary schools in Hong Kong. Time spent in ST $(<100$ counts per minute [cpm]), moderate-to-vigorous PA (MVPA), and light-intensity PA (LPA) were measured by accelerometer at baseline and then at 1-year and 2-year follow-ups. Mean annual changes were determined using mixed-effects linear models for children who provided 3-day valid data (including 1 weekend day) for at least two time points $(n=412)$. Magnitude of changes between weekdays and weekends was compared using age $\times$ time-segment interactions.

Results: At each assessment wave, the percentage of time spent in MVPA (\% MVPA) and LPA (\% LPA) was consistently high, whereas the percentage of time spent in ST (\% ST) was lower on weekends than weekdays. A decrease in \% MVPA was found for both weekdays (mean annual change: boys, $-0.7,95 \% \mathrm{Cl}=-0.9$ to -0.1 ; girls, -0.8 , $95 \% \mathrm{Cl}=-1.0$ to -0.6 ) and weekends (boys, $-1.2,95 \% \mathrm{Cl}=-1.5$ to $-0.9 ;$ girls, $-1.4,95 \% \mathrm{Cl}=-1.6$ to -1.1 ). An increase was found in \% ST for both weekdays (boys, $1.3,95 \% \mathrm{Cl}=0.7$ to $1.9 ;$ girls, $2.4,95 \% \mathrm{Cl}=1.9$ to 3.3 ) and weekends (boys, $1.8,95 \% \mathrm{Cl}=1.1$ to $2.5 ;$ girls, $2.6,95 \% \mathrm{Cl}=1.9$ to 3.3). Mean annual change in MVPA time (min) was greater on weekends than weekdays (difference: boys, $3.0,95 \% \mathrm{Cl}=0.3$ to 5.7 ; girls, $3.5,95 \% \mathrm{Cl}=1.1$ to 5.8 ).

Conclusions: Age-related decline in MVPA was more marked on weekends than weekdays. Interventions to hinder age-related changes in PA and ST should target both time segments, but weekends warrant particular attention for interventions targeting PA maintenance due to the greater declines.
\end{abstract}

Keywords: Children, Moderate-to-vigorous physical activity, Sedentary behaviour, Longitudinal, Time segments, Accelerometry

\footnotetext{
* Correspondence: wendyhuang@hkbu.edu.hk

${ }^{2}$ Department of Physical Education, AAB924, Academic and Administration

Building, Hong Kong Baptist University, Kowloon Tong, Hong Kong, China

Full list of author information is available at the end of the article
} 


\section{Background}

Physical activity (PA) is associated with numerous health benefits in children [1]. In addition, excessive sedentary behaviour (particularly when measured as screen-based behaviours) has emerged as a risk factor for cardiometabolic disease independent of moderate-to-vigorous PA (MVPA) [2, 3]. The generally accepted public guidelines recommend at least 60 min per day of MVPA and reducing screen time to less than $2 \mathrm{~h}$ a day for school-aged youth [4]. However, many children do not achieve these recommendations, and age-related decline in PA [5] and increase in sedentary time (ST) [6] during childhood and adolescence have been documented in recent systematic reviews. PA declines by $7 \%$ every year during adolescence [5], and objectively assessed daily ST increases by 30 min each year during childhood and adolescence [6]. It was noteworthy that current evidence with regard to longitudinal changes in PA and ST is mainly from western countries.

Many studies have focused on overall PA, but PA levels may vary according to specific time segments of the week. For school-aged children, notable differences in PA levels have been found between weekdays and weekends. A meta-analysis of cross-sectional data showed that children aged 4-18 years spent approximately 14 more minutes per day on PA during weekdays than weekends [7]. The authors anticipated that children have autonomy during weekends and generally do not choose to participate in health-enhancing PA [7]. However, data in this meta-analysis neither accounted for the total time the children wore the accelerometer nor investigated age-related differences. Few studies have compared the magnitude of longitudinal changes in PA and ST between weekdays and weekends, and the findings are equivocal. Brooke et al. [8] reported that for 10year-old children MVPA declined during both weekdays and weekends over a 4-year follow-up period, but the annual change in MVPA was greater on weekends than weekdays. However, adolescents participating in the PEACH project had relatively stable PA levels from 12 to 15 years of age but accumulated more time in sedentary pursuits during both weekdays and weekends [9]. Furthermore, the magnitude of change in both ST and PA did not differ between school days and weekends [9].

Comparing the magnitude of behavioural change between school days and weekends may be helpful in suggesting intervention strategies to hinder the age-related decline in PA and increase in ST. It may be particularly important for Chinese children for two reasons. First, previous research has suggested weekends to be the promising target for PA maintenance due to the greater age-related declines. Contrary to what has been observed in Caucasian children, a survey conducted in Hong Kong [10] suggests that Chinese youth are more physically active on weekends than weekdays. It remains unknown on whether the difference persists with age. Second, previous studies conducted in Chinese children have been cross-sectional and used subjective measurement of PA $[10,11]$, which has limited accuracy and precision in estimating the amount and intensity of PA [12]. Therefore, the purposes of this study were to describe the 2-year changes in accelerometer-assessed PA and ST among Chinese children and to examine if the magnitude of the changes differed between weekdays and weekends.

\section{Methods}

\section{Study design and participants}

Data were from the Understanding Children's Activity and Nutrition (UCAN) study, which was a 3-year longitudinal study investigating determinants of PA and sedentary behaviour in Chinese children in Hong Kong. Ethical approval for this study was obtained from the Survey and Behavioural Research Ethics Committee of the Chinese University of Hong Kong. Primary schools located in different districts were purposely sampled between June and August 2009 to represent a variety of socio-economic status (SES). Among the total of 18 districts, 3 districts with low, medium, or high SES (according to the median domestic household income) were randomly selected. Within each of the selected district, invitations were sent to half of the primary schools. Initially, 27 schools agreed to participate in the study, but 3 of them later dropped out for school-related reasons leaving 24 schools (5 from high SES, 6 from medium SES and 13 from low SES) included (24\% of invited). Written parental consent forms were sent to 6 randomly selected classes in grades 1 to 3 from each participating school. Finally, parental consent was sought from 1666 grades 1-3 children from 24 schools (42\% response rate). The parents of all the participating children were asked to complete a questionnaire for both determinants and PA outcomes [13, 14], and only a subsample of them agreed to their child wearing an accelerometer. The limited number of ActiGraph accelerometers $(n=$ 40) that were available for use at the beginning of the study meant that accelerometer data were only collected at baseline from 448 children (26.9 \% of the whole sample and $43.8 \%$ of those whose parents had agreed), but at the 1-year and 2-year follow-ups data were collected for all the children whose parents had agreed. The details of numbers at each time point are shown in Table 1. No differences were found in sex or body mass index (BMI) between those who wore the accelerometers and those who did not. However, the children who wore the accelerometers were older and had parents of lower educational attainment than those who did not wear the 
Table 1 Characteristics of the sample at the three time points

\begin{tabular}{|c|c|c|c|c|}
\hline & Baseline & 1-yr follow-up & 2-yr follow-up & $\geq 2$ time points $^{\mathrm{a}}$ \\
\hline $\mathrm{N}$ consented to wear ActiGraph/distributed & $1023 / 448$ & $874 / 874$ & $706 / 706$ & NA \\
\hline $\mathrm{N}$ with returned ActiGraph & 445 & 852 & 693 & NA \\
\hline $\begin{array}{l}N \text { with } \geq 3 \text { days of PA data including } 1 \text { weekend day } \\
\text { (\% of distributed sample) }\end{array}$ & $263(58.7)$ & $537(61.4)$ & $421(59.6)$ & 412 \\
\hline Age (yrs) & $7.8(1.0)$ & $8.6(1.0)$ & $9.5(1.0)$ & $7.6(1.0)$ \\
\hline Gender (\% boys) & 52.6 & 52.8 & 54.8 & 54.0 \\
\hline $\mathrm{BMI}\left(\mathrm{kg} \cdot \mathrm{m}^{-2}\right)$ & $17.3(3.1)$ & $17.7(3.3)$ & $18.2(3.5)$ & $17.1(3.0)$ \\
\hline Overweight and obese (\%) & 25.8 & 24.6 & 26.2 & 24.1 \\
\hline Parental education level & $4.3(1.4)$ & $4.5(1.5)$ & $4.5(1.5)$ & $4.5(1.5)$ \\
\hline Having sibling (\%) & 62.7 & 64.9 & 65.2 & 66.1 \\
\hline Marital status (\% single parent) & 12.9 & 10.4 & 10.4 & 8.8 \\
\hline Wearing time $\left(\min \cdot d^{-1}\right)$ & $789(90)$ & $796(78)$ & $801(90)$ & $793(86)$ \\
\hline $\operatorname{MVPA}\left(\min \cdot d^{-1}\right)$ & $61.5(23.3)$ & $50.0(20.4)$ & $45.6(20.7)$ & $64.2(22.7)$ \\
\hline $\operatorname{LPA}\left(\min \cdot d^{-1}\right)$ & $323.2(67.3)$ & $329.6(62.4)$ & $314.8(64.4)$ & $326.5(65.8)$ \\
\hline ST $\left(\min \cdot d^{-1}\right)$ & $402.1(73.2)$ & $414.7(72.3)$ & $439.8(80.1)$ & $402.9(72.5)$ \\
\hline$\%$ MVPA & $8.2(3.1)$ & $6.4(2.6)$ & $5.9(2.8)$ & $8.4(3.0)$ \\
\hline$\%$ LPA & $40.8(6.6)$ & $41.0(6.4)$ & $38.8(6.9)$ & $40.8(6.5)$ \\
\hline$\%$ ST & $51.0(8.1)$ & $52.5(7.6)$ & $55.2(8.3)$ & $50.7(7.9)$ \\
\hline
\end{tabular}

Values are means (SD) unless otherwise specified; MVPA moderate-to-vigorous physical activity; LPA, light-intensity physical activity; ST sedentary time; BMI body mass index

${ }^{a}$ For those who provided valid accelerometer data for at least two time points $(N=412)$, baseline data are presented

accelerometer. The current analysis was restricted to accelerometer-assessed activity outcomes.

Baseline data (T1) were collected in two school semesters (September to November 2009 and January to April 2010). To minimise seasonal variations, follow-up measurements 1 year (T2) and 2 years (T3) later were conducted as close to the baseline time of year as practicable. Anthropometric data were collected during school visits by trained assessors. Meanwhile, ActiGraph accelerometers and questionnaires were distributed to the children and their parents.

\section{Measurement of physical activity and sedentary time}

The children were each instructed to wear an ActiGraph GT3X accelerometer (ActiGraph, Pensacola, Florida, USA), which was attached to an elasticised belt worn at hip level for 8 consecutive days. The accelerometer was only removed during swimming, showering and sleeping. A 1-min epoch was set to record data. Three days of accelerometer data (including 1 weekend day) with a minimum of $10 \mathrm{~h}$ ' recording per day were considered to be valid $[8,15]$. Accelerometer data were downloaded using ActiLife 6, and screened and analysed using MeterPlus software (Santech Inc., V.4.3, http://www.meterplussoftware.com). Age-specific cutoff counts [16] were applied to quantify time spent in MVPA ( $\geq 4$ METs; a 4 MET intensity represents 4 times resting energy expenditure) [17] and light-intensity PA
(LPA). For example, the cut-off point for 4 METs corresponded to $1910 \mathrm{cpm}$ for 10-year-old children. A threshold of $<100 \mathrm{cpm}$ was used to define ST [18]. After accounting for total wearing time at different time points, three constructs were generated: MVPA, expressed as a percentage of daily wearing time spent in MVPA (\% MVPA); LPA, expressed as a percentage of daily wearing time spent in LPA (\% LPA) and ST, expressed as a percentage of daily wearing time spent in sedentary behaviours (\% ST). Daily minutes in different intensities of PA and ST were also calculated. These outcome measures were derived for the whole week and separately for weekdays and weekends. Crosssectional comparisons between weekdays and weekends were conducted for children who provided valid accelerometer data for at least 2 weekdays and 1 weekend day at each time point $(n=263$ at T1, $n=537$ at T2, and $n=421$ at T3). Overall, 412 children (222 boys) with valid accelerometer data from at least two time points were included in the analysis to compare the mean annual changes for weekdays and weekends.

\section{Anthropometric and socio-demographic factors}

The children were weighed in the minimum clothing possible and they were measured for height while standing without shoes. Their BMIs were subsequently calculated by dividing their weight $(\mathrm{kg})$ by their height squared $\left(\mathrm{m}^{2}\right)$. Overweight and obesity were classified 
according to the international standard for children using age- and sex-specific cut-off points [19]. The demographic information was reported by parents, including the responding parent's age, sex, educational attainment, marital status, the number of siblings, and the child's sex and date of birth.

\section{Statistical analyses}

The differences in baseline socio-demographic characteristics between the children with valid data from at least two time points and the remaining baseline sample were tested using $t$ tests. The participants' characteristics were also compared at different time points. Mean values (standard deviations) were calculated for the outcome measures because they were approximately normally distributed.

The cross-sectional comparisons at each time point between weekdays and weekends were determined using paired $t$ tests. The mean annual changes in the outcomes were determined using linear mixed models controlling for time-vary BMI, parental educational attainment, marital status, sibling numbers and wearing time (for absolute minutes). To account for the clustering by school, a random intercept for the variable 'school' was included in the models. Each outcome (MVPA, LPA and ST) was modelled separately. The gender differences in all of the outcomes and gender-age interactions were found in LPA and ST, and the mixed models were therefore separated by gender for the outcomes. Weekday-weekend comparisons in mean annual change were determined by using an age $\mathrm{x}$ time-segment interaction term. The statistical analyses were performed using SPSS 22.0 and a probability level of 0.05 was used.

\section{Results}

The socio-demographic characteristics of the sample at different time points were compared and no differences were found in parental educational level, number of siblings or marital status (Table 1). Compared with the baseline sample, the children who provided valid data for at least two time points were more likely to have siblings and less likely to have single parents. However, no differences were found in activity outcomes between these groups.

Table 2 shows the cross-sectional comparisons of MVPA, LPA and ST between weekdays and weekends. At each assessment wave, wearing times were longer on weekdays than weekends. Whereas \% MVPA and \% LPA were higher on weekends than weekdays, \% ST was lower. With regard to absolute MVPA minutes, timesegment differences were only found at baseline (weekdays vs weekends: $61 \pm 24 \mathrm{~min} /$ day vs $70 \pm 37 \mathrm{~min} /$ day, $p<0.05)$, not at T2 or T3.

The comparative mean annual changes in MVPA, LPA and ST are shown in Table 3. Whereas \% MVPA and \% LPA declined on both weekdays and weekends, \% ST increased. The daily declines in MVPA minutes ranged from $6 \mathrm{~min}$ for weekdays in boys to $10 \mathrm{~min}$ for weekends in girls. The mean annual increases in ST were $19 \mathrm{~min} /$ day in girls and 11-13 $\mathrm{min} /$ day in boys. The magnitude of annual change in \%MVPA was greater on weekends than weekdays for both boys $(B=0.5,95 \%$ $\mathrm{CI}=0.1$ to 0.9$)$ and girls $(B=0.6,95 \% \mathrm{CI}=0.2$ to 0.9$)$. No differences in magnitude of change were found in LPA and ST.

\section{Discussion}

This study examined longitudinal changes of objectively assessed PA and ST over a 2-year period among Chinese children in Hong Kong. Consistent to the findings from the cross-sectional survey in Hong Kong [10], Chinese children accumulated more MVPA time and spent less time in ST on weekends than weekdays and this pattern persisted for two consecutive years. MVPA and LPA

Table 2 Cross-sectional comparisons of MVPA, LPA and ST between weekdays and weekends at three time points

\begin{tabular}{|c|c|c|c|c|c|c|}
\hline & \multicolumn{2}{|c|}{$\mathrm{T} 1(n=263)$} & \multicolumn{2}{|c|}{$\mathrm{T} 2(n=537)$} & \multicolumn{2}{|c|}{ T3 $(n=421)$} \\
\hline & Weekday & Weekend & Weekday & Weekend & Weekday & Weekend \\
\hline Wearing time $\left(\min \cdot d^{-1}\right)$ & $816(90)$ & $738(132)$ & $828(84)$ & $726(98)$ & $834(96)$ & $732(126)$ \\
\hline \multicolumn{7}{|l|}{ Daily time in minutes } \\
\hline MVPA $\left(\min \cdot d^{-1}\right)$ & $61(24)$ & $70(37)$ & $51(21)$ & $53(34)$ & $46(21)$ & $48(33)$ \\
\hline LPA $\left(\min \cdot d^{-1}\right)$ & $329(74)$ & $307(76)$ & $336(68)$ & $311(75)$ & $322(70)$ & $297(79)$ \\
\hline $\mathrm{ST}\left(\min \cdot \mathrm{d}^{-1}\right)$ & $420(79)$ & $356(108)$ & $436(79)$ & $360(91)$ & $463(86)$ & $380(107)$ \\
\hline \multicolumn{7}{|c|}{ Proportion of time (\% of wearing time) } \\
\hline$\%$ MVPA & $7.6(2.9)$ & $9.7(5.3)$ & $6.2(2.6)$ & $7.3(4.6)$ & $5.6(2.5)$ & $6.7(4.8)$ \\
\hline$\%$ LPA & $40.4(7.0)$ & $42.0(7.8)$ & $40.8(6.7)$ & $43.0(8.2)$ & $38.7(6.9)$ & $41.0(8.8)$ \\
\hline$\%$ ST & $52.0(8.0)$ & $48.3(10.5)$ & $53.0(8.0)$ & 49.7 (10.2) & $55.7(7.9)$ & $52.3(11.2)$ \\
\hline
\end{tabular}

Values are means (SD); MVPA, moderate-to-vigorous physical activity; LPA light-intensity physical activity; ST sedentary time All differences between weekdays and weekend days are significant $(p<0.05)$ except for MVPA $\left(\min ^{-1} \mathrm{~d}^{-1}\right)$ at T2 and T3 No gender-outcome interactions were found; the results were combined for both boys and girls 
Table 3 Mean and comparative annual change in MVPA, LPA and ST at weekdays and weekends

\begin{tabular}{|c|c|c|c|}
\hline & Weekday $^{a}$ & Weekend $^{\mathrm{a}}$ & Difference in annual change ${ }^{\mathrm{b}}$ (reference: weekend) \\
\hline \multicolumn{4}{|l|}{ Boys } \\
\hline MVPA $\left(\min \cdot d^{-1}\right)$ & $-5.5(-7.3,-3.6)$ & $-8.5(-10.6,-6.3)$ & $3.0(0.3,5.7)$ \\
\hline $\operatorname{LPA}\left(\min \cdot d^{-1}\right)$ & $-5.8(-9.4,-2.2)$ & $-4.2(-8.6,-0.1)$ & $-1.5(-7.0,3.9)$ \\
\hline ST $\left(\min \cdot d^{-1}\right)$ & $11.2(6.8,15.5)$ & $12.7(7.4,18.0)$ & $-1.5(-8.1,5.1)$ \\
\hline$\%$ MVPA & $-0.7(-0.9,-0.1)$ & $-1.2(-1.5,-0.9)$ & $0.5(0.1,0.9)$ \\
\hline$\%$ LPA & $-0.7(-1.1,-0.2)$ & $-0.6(-1.2,-0.0)$ & $-0.0(-0.8,0.7)$ \\
\hline$\%$ ST & $1.3(0.7,1.9)$ & $1.8(1.1,2.5)$ & $-0.5(-1.3,0.4)$ \\
\hline \multicolumn{4}{|l|}{ Girls } \\
\hline MVPA $\left(\min \cdot d^{-1}\right)$ & $-6.5(-8.1,-4.9)$ & $-10.0(-11.9,-8.1)$ & $3.5(1.1,5.8)$ \\
\hline LPA $\left(\min \cdot d^{-1}\right)$ & $-12.8(-16.6,-8.9)$ & $-9.3(-13.9,-4.8)$ & $-3.5(-9.2,2.3)$ \\
\hline ST $\left(\min \cdot d^{-1}\right)$ & $19.3(14.8,23.8)$ & $19.3(14.0,24.6)$ & $-0.0(-6.6,6.7)$ \\
\hline$\%$ MVPA & $-0.8(-1.0,-0.6)$ & $-1.4(-1.6,-1.1)$ & $0.6(0.2,0.9)$ \\
\hline$\%$ LPA & $-1.6(-2.1,-1.1)$ & $-1.2(-1.8,-0.6)$ & $-0.4(-1.1,0.4)$ \\
\hline$\%$ ST & $2.4(1.9,3.3)$ & $2.6(1.9,3.3)$ & $-0.2(-1.0,0.7)$ \\
\hline
\end{tabular}

Data are presented in $\beta$ coefficients and $95 \%$ confidence intervals

MVPA moderate-to-vigorous physical activity; LPA light-intensity physical activity; ST sedentary time

${ }^{a}$ Results from linear mixed models for children with valid data for at least 3 days including 1 weekend day $\geq 2$ time points $(n=412) ; \beta$ coefficients for weekdays and weekends indicate mean annual change

${ }^{b}$ Comparative annual change between weekday and weekend determined by linear mixed models. $\beta$ coefficients represent the difference in change between weekday and weekend for every 1-year increase in age. The significant results are in bold. Models were adjusted for time-varying BMI, parental education, marital status, number of siblings, and wearing time (for absolute minutes)

declined, and ST increased, on both weekdays and weekends over a 2-year period. Greater declines for MVPA were observed on weekends compared with weekdays. These findings suggest that both weekdays and weekends need to be targeted for interventions to minimise age-related changes in PA and ST for Chinese children. Weekends may be particular important for hindering age-related decline.

This is the first longitudinal study to examine changes in time-segment-specific PA and ST in Chinese children in Hong Kong. The findings on the differences between weekdays and weekends were consistent with the crosssectional survey in Hong Kong showing that young people seemed to be more physically active on weekends than weekdays [10]. However, opposite results have been found in other countries $[8,20,21]$. As children have more discretionary time during weekends than school days, it is anticipated that they are more likely to choose sedentary activities rather than PA on weekends. A recent systematic review and meta-analysis [7] showed a consistent trend of higher MVPA on weekends than weekdays for school-aged children and adolescents. The authors assumed that the length of weekday and weekend time segments, i.e. the total wearing time of the accelerometer, was comparable [7], but this was not the case for the Chinese children. Children in Hong Kong usually sleep longer and get up later on weekends [22], so the participants had a shorter time to wear the accelerometer. Interestingly, they managed to accumulate more MVPA time despite the shorter wake period on weekends. The results indicated that Chinese children may experience various barriers to participating in PA on weekdays. Recess, physical education (PE) classes, lunchtime and after-school periods are potential PA time periods for children. Although PE is required for schoolaged children in Hong Kong, the actual lesson length is $30 \%$ shorter than scheduled and less than half the time is spent in MVPA [23]. In addition, study-related activities, such as homework, occupy most Chinese children's after-school time [24]. For safety reasons, many schools do not allow their students to play outdoors during recess and lunchtime. This may explain why children spend less time in MVPA and more time in sedentary activities during weekdays.

The age-related decline in PA and increase in ST in Chinese children are in line with previous research [25-28]. Both intensity levels of PA were replaced by ST over the 2-year period. The magnitude of changes was even greater than that previously reported for older children and adolescents. Corder et al. [28] reported an annual increase of $10.6 \mathrm{~min} /$ day in ST and a parallel reduction of $3 \mathrm{~min} /$ day in MVPA and $9.8 \mathrm{~min} /$ day in LPA among 9-10-year-old children. During adolescence, MVPA tends to remain relatively stable, but ST continues to increase at the expense of a reduction in LPA [9]. Children in the current study were in an early primary school year and on average they met the PA recommendations of $60 \mathrm{~min} /$ day at baseline. At follow- 
ups, their mean daily MVPA dropped below the recommendations. Survey studies in Hong Kong have shown that fewer than $50 \%$ of grades 4-6 children achieve the PA recommendations [11,29] and the prevalence is even lower for adolescents [10]. Such behavioural changes in early childhood may have adverse effects on cardiometabolic health. According to a meta-analysis of multiple cohort studies [30], a 10-min increase in MVPA per day is associated with lower systolic blood pressure, fasting insulin and triglycerides, whereas a 60-min increase in ST leads to higher fasting insulin. Furthermore, the combined association of MVPA and ST with metabolic factors indicated that a difference of $20 \mathrm{~min} /$ day in MVPA was associated with 5.6 more $\mathrm{cm}$ in waist circumference in children and adolescents [30]. In addition, recent studies have shown that LPA is also important for health. Kwon at al. [31] found that accelerometer-assessed LPA was negatively associated with body fat mass measured by dual energy X-ray absorptiometry among older children aged 8 and 11 years while controlling for MVPA. These findings indicate the urgency of interventions focusing on PA maintenance implemented at an early stage and targeting all intensity levels of PA and sedentary activities.

A difference in magnitude of change between weekdays and weekends was only found in MVPA, not in ST or LPA. Not many longitudinal studies have compared time-segment-specific changes in PA or ST. A greater reduction in MVPA on weekends than weekdays is consistent with the study by Brooke et al. [8], which showed that both total PA (in accelerometer cpm) and MVPA minutes declined to a greater extent on weekends compared with weekdays among British children aged 10 years at baseline. However, ST was not examined in Brooke et al.'s study. Furthermore, British children were more physically active during weekdays than weekends. Identifying the most promising time periods during which to change children's activity behaviours is believed to be useful for informing future interventions. The lower levels of PA [7] and greater reduction in MVPA on weekends [8] have led some researchers to suggest that the weekend may be a promising time period for interventions to minimise age-related changes in ST and PA. It is noteworthy that although the reduction in PA was substantial at weekends, the Chinese children still managed to accumulate similar or even more time in MVPA within a shorter wake period compared with weekdays at each time point. A better understanding of the factors influencing PA on weekdays and weekends may be helpful to explain this phenomenon. It has been suggested that predictors of 1-year change in PA were different for weekdays and weekends [32]. Parent support was associated with less decline in weekend PA whereas peer support was important in maintaining weekday PA [32]. Both parent and peer support have been found to be associated with PA and screen-based behaviours in children in Hong Kong [29]. Whether the relationships differ between weekdays and weekends need further investigation. In addition, children's participation in PA is more likely to be influenced by the neighbourhood environment that is unique to Hong Kong [33]. Qualitative research may also be useful to explore the reasons for the higher PA levels among Chinese children on weekends.

This study has several limitations. Higher response rate was sought from schools in districts with low SES compared with medium and high SES. As a result, half of the participants were from low SES areas. Furthermore, children who had parents with lower education levels were more likely to agree to wear the accelerometer. As a result, the findings should be generalised to other children with caution. There is inconsistent evidence for cross-sectional associations of SES with PA in children and adolescents, including in Hong Kong [29]. The longitudinal influences of SES on PA and ST need further investigation. There is no universal consensus on accelerometer cut points for determining PA. The MVPA minutes reported are therefore inevitably influenced by the cut points applied. The use of the relative lower cut-off counts may partly explain the higher prevalence of Chinese children meeting PA recommendations. However, the pattern for longitudinal changes will not be affected. To make weekday-weekend comparisons, 3 valid days of accelerometer data, including 1 weekend day, were required, which reduced the sample size. Compliance in wearing an accelerometer has been found to be more difficult on weekends than on weekdays. In the current study, over $80 \%$ of the sample monitored provided valid accelerometer data on at least 3 weekdays at each assessment wave; however, only $60 \%$ of them met the weekend wearing-time criteria. This compliance rate is similar or even higher than that reported in previous studies [9, 17]. Retention strategies, specific for weekends, warrant further research. Although using one weekend day is a commonly applied inclusive criterion for accelerometer data [8,9], it may allow for atypical weekend activity events to influence the results. However, the proportion of children who provided valid data for only one weekend day was less than $30 \%$ at each time point; thus may not have significant influence on the results. Finally, comparisons with other studies using shorter epoch for the accelerometer should be cautious since the epoch may have impact on the estimated PA levels for children [34].

\section{Conclusions}

Chinese children were more physically active on weekends than weekdays. An age-related decline in MVPA 
was more marked on weekends than weekdays. Interventions to hinder age-related decline in PA and increase in ST should target both time segments, but weekends warrant particular attention for interventions targeting PA maintenance due to the greater declines of PA.

\section{Abbreviations}

BMI: Body mass index; PA: Physical activity; MVPA: Moderate-to-vigorous physical activity; LPA: Light-intensity physical activity; ST: Sedentary time; UCAN: Understanding Children's Activity and Nutrition.

\section{Competing interests}

The authors declare that they have no competing interests.

\section{Authors' contributions}

SW and WH conceived and designed the study. WH and GH carried out the data collection. WH drafted the initial manuscript and conducted the statistical analyses. All authors critically reviewed the draft manuscript. All authors read and approved the final manuscript.

\section{Acknowledgements}

The UCAN study was supported by the General Research Fund (GRF) from the Research Grants Council (RGC) of the Government of the Hong Kong Special Administrative Region, China (GRF 451308). The authors are grateful to all the children and their parents who participated in this study, along with the school teachers and student helpers who assisted in the UCAN project. We also thank Dr David Wilmshurst, from the Chinese University of Hong Kong, for editing the manuscript.

\section{Author details}

'Department of Sports Science and Physical Education, The Chinese University of Hong Kong, Shatin, Hong Kong, China. ${ }^{2}$ Department of Physical Education, AAB924, Academic and Administration Building, Hong Kong Baptist University, Kowloon Tong, Hong Kong, China.

Received: 12 August 2015 Accepted: 16 December 2015 Published online: 29 December 2015

\section{References}

1. Janssen I, Leblanc AG. Systematic review of the health benefits of physical activity and fitness in school-aged children and youth. Int J Behav Nutr Phys Act. 2010;7:40

2. Saunders TJ, Chaput JP, Tremblay MS. Sedentary behaviour as an emerging risk factor for cardiometabolic diseases in children and youth. Can J Diabetes. 2014, 38(1):53-61.

3. Coombs NA, Stamatakis E. Associations between objectively assessed and questionnaire-based sedentary behaviour with BMI-defined obesity among general population children and adolescents living in England. BMJ Open. 2015;5(6):e007172

4. Tremblay MS, Leblanc AG, Janssen I, Kho ME, Hicks A, Murumets K, et al. Canadian sedentary behaviour guidelines for children and youth. Appl Physiol Nutr Metab. 2011;36(1):59-64. 5-71.

5. Dumith SC, Gigante DP, Domingues MR, Kohl 3rd HW. Physical activity change during adolescence: a systematic review and a pooled analysis. Int J Epidemiol. 2011:40(3):685-98.

6. Tanaka C, Reilly JJ, Huang WY. Longitudinal changes in objectively measured sedentary behaviour and their relationship with adiposity in children and adolescents: systematic review and evidence appraisal. Obes Rev. 2014;15(10):791-803.

7. Brooke $\mathrm{HL}$, Corder $\mathrm{K}$, Atkin AJ, van Sluijs EM. A systematic literature review with meta-analyses of within- and between-day differences in objectively measured physical activity in school-aged children. Sports Med. 2014;44(10): 1427-38.

8. Atkin AJ, Corder K, Ekelund U, van Sluijs EM. Changes in time-segment specific physical activity between ages 10 and 14 years: A longitudinal observational study. J Sci Med Sport. 2014; doi:10.1016/j.jsams.2014.10.003

9. Harding SK, Page AS, Falconer C, Cooper AR. Longitudinal changes in sedentary time and physical activity during adolescence. Int J Behav Nutr Phys Act. 2015;12(1):44
10. Mak KK, Ho SY, Lo WS, McManus AM, Lam TH. Prevalence of exercise and non-exercise physical activity in Chinese adolescents. Int J Behav Nutr Phys Act. 2011;8:3.

11. Lam JW, Sit CH, Cerin E. Physical activity and sedentary behaviours in Hong Kong primary school children: Prevalence and gender differences. Prev Med. 2010:51(1):96-7.

12. Reilly JJ, Penpraze V, Hislop J, Davies G, Grant S, Paton JY. Objective measurement of physical activity and sedentary behaviour: review with new data. Arch Dis Child. 2008:93(7):614-9.

13. Huang YJ, Wong SH, Salmon J. Reliability and validity of the modified Chinese version of the Children's Leisure Activities Study Survey (CLASS) questionnaire in assessing physical activity among Hong Kong children. Pedia Exerc Sci. 2009:21(3):339-53.

14. Huang YJ, Wong SH, Salmon J, Hui SS. Reliability and validity of psychosocial and environmental correlates measures of physical activity and screen-based behaviors among Chinese children in Hong Kong. Int J Behav Nutr Phys Act. 2011:8:16

15. Ruiz JR, Ortega FB, Martinez-Gomez D, Labayen I, Moreno LA, De Bourdeaudhuij I, et al. Objectively measured physical activity and sedentary time in European adolescents: the HELENA study. Am J Epidemiol. 2011;174(2):173-84.

16. Trost SG, Pate RR, Sallis JF, Freedson PS, Taylor WC, Dowda M, et al. Age and gender differences in objectively measured physical activity in youth. Med Sci Sports Exerc. 2002;34(2):350-5.

17. Arundell L, Ridgers ND, Veitch J, Salmon J, Hinkley T, Timperio A. 5-year changes in afterschool physical activity and sedentary behavior. Am J Prev Med. 2013:44(6):605-11.

18. Kim Y, Lee JM, Peters BP, Gaesser GA, Welk GJ. Examination of different accelerometer cut-points for assessing sedentary behaviors in children. PLoS One. 2014;9(4):e90630

19. Cole TJ, Bellizzi MC, Flegal KM, Dietz WH. Establishing a standard definition for child overweight and obesity worldwide: international survey. BMJ. 2000; 320(7244):1240-3

20. Rowlands AV, Pilgrim EL, Eston RG. Patterns of habitual activity across weekdays and weekend days in 9-11-year-old children. Prev Med. 2008; 46(4):317-24

21. Fairclough SJ, Boddy LM, Mackintosh KA, Valencia-Peris A, Ramirez-Rico E. Weekday and weekend sedentary time and physical activity in differentially active children. J Sci Med Sport. 2015;18(4):444-9.

22. Wing YK, Li SX, Li AM, Zhang J, Kong AP. The effect of weekend and holiday sleep compensation on childhood overweight and obesity. Pediatrics. 2009;124(5):e994-e1000.

23. Chow BC, McKenzie TL, Louie L. Children's physical activity and environmental influences during elementary school physical education. J Teach Phys Educ. 2008;27(1):38-50

24. Huang WY, Wong SH. Time use clusters in children and their associations with sociodemographic factors. Journal of Public Health. 2015; doi:10.1093/pubmed/fdv088

25. Belanger M, Gray-Donald K, O'Loughlin J, Paradis G, Hanley J. When adolescents drop the ball: sustainability of physical activity in youth. Am J Prev Med. 2009;37(1):41-9.

26. Kjonniksen $\mathrm{L}$, Torsheim T, Wold B. Tracking of leisure-time physical activity during adolescence and young adulthood: a 10-year longitudinal study. Int J Behav Nutr Phys Act. 2008:5:69.

27. Basterfield L, Adamson AJ, Frary JK, Parkinson KN, Pearce MS, Reilly JJ. Longitudinal study of physical activity and sedentary behavior in children. Pediatrics. 2011;127(1):e24-30.

28. Corder K, Sharp SJ, Atkin AJ, Griffin SJ, Jones AP, Ekelund U et al. Change in objectively measured physical activity during the transition to adolescence. Br J Sports Med. 2014:doi:10.1136/bjsports-2013-093190.

29. Huang WY, Wong SH, Salmon J. Correlates of physical activity and screenbased behaviors in Chinese children. J Sci Med Sport. 2013;16(6):509-14.

30. Ekelund U, Luan J, Sherar LB, Esliger DW, Griew P, Cooper A. Moderate to vigorous physical activity and sedentary time and cardiometabolic risk factors in children and adolescents. JAMA. 2012:307(7):704-12.

31. Kwon S, Janz KF, Burns TL, Levy SM. Association between light-intensity physical activity and adiposity in childhood. Pediatr Exerc Sci. 2011;23(2): 218-29.

32. Corder K, Craggs C, Jones AP, Ekelund U, Griffin SJ, van Sluiis EM. Predictors of change differ for moderate and vigorous intensity physical activity and for weekdays and weekends: a longitudinal analysis. Int J Behav Nutr Phys Act. 2013;10:69. 
33. He G, Cerin E, Huang WY, Wong SH. Understanding neighborhood environment related to Hong Kong children's physical activity: a qualitative study using nominal group technique. PLoS One. 2014;9(9): e106578.

34. Edwardson CL, Gorely T. Epoch length and its effect on physical activity intensity. Med Sci Sports Exerc. 2010;42(5):928-34.

Submit your next manuscript to BioMed Central and we will help you at every step:

- We accept pre-submission inquiries

- Our selector tool helps you to find the most relevant journal

- We provide round the clock customer support

- Convenient online submission

- Thorough peer review

- Inclusion in PubMed and all major indexing services

- Maximum visibility for your research

Submit your manuscript at www.biomedcentral.com/submit 